\title{
Streamline-Based Microfluidic Devices for Erythrocytes and Leukocytes Separation
}

\author{
Siyang Zheng, Jing-Quan Liu, and Yu-Chong Tai, Fellow, IEEE
}

\begin{abstract}
In this paper, we report two devices for the continuous size-based separation of particles, such as blood cells, which is an important step for on-chip blood preparation. Unlike previously demonstrated passive fluidic devices for particle separation, the local geometry of the bifurcated side channels was used as a design parameter. The design of the devices was based on 2-D fluidic simulation of a T-shaped model. This novel approach was proved to be effective in predicting device performance. The critical particle size for separation was clearly defined in the bifurcated region by simulation under the established theoretical framework. We validated the operation principle of the devices by separating 5- and $10-\mu \mathrm{m}$ polystyrene beads. Human leukocytes were also successfully separated from erythrocytes with $97 \%$ efficiency. The separation region of the device had a small footprint for the separation of particles in micrometer range, which makes this device a good candidate to be integrated into a lab-on-a-chip system. The particles were collected in different exit channels after they were separated, which facilitated further sensing and processing. Similar to cross-flow filters, particles were separated perpendicular to the flow direction. The filtering effect was achieved with the collection zones established by the fluidic field. Clogging was minimized by designing the minimal channel width of the devices larger than the largest particle diameter. Solvent exchange could be accomplished for particles.

[2007-0196]
\end{abstract}

Index Terms-Blood, microelectromechanical devices.

\section{INTRODUCTION}

$\mathbf{T}$ HE DEVELOPMENT of micrototal-analysis (microTAS) and lab-on-a-chip technologies represents a valuable tool for biological cell study and clinical applications involving cells. The ability to isolate homogeneous and concentrated cell populations from heterogeneous cell mixtures is a prerequisite for understanding cell development and differentiation, the underlying biochemistry of specific cell types, and cell responses to environmental stimuli [1], [2]. Blood cells form a heterogeneous mixture in nature and continuously respond to the physiological and pathological changes of human body. Thus, they are believed to be able to provide rapid and accurate diagnostic and prognostic capabilities for medical situations, such

Manuscript received August 5, 2007; revised February 8, 2008. First published July 18, 2008; last published August 1, 2008 (projected). This work was supported by the National Space Biomedical Research Institute through NASA NCC 9-58. Subject Editor A. Lee.

S. Zheng and Y.-C. Tai are with the Department of Electrical Engineering, California Institute of Technology, Pasadena, CA 91125 USA (e-mail: siyang@mems.caltech.edu).

J.-Q. Liu is with Department of Electrical Engineering, California Institute of Technology, Pasadena, CA 91125 USA, and also with the Research Institute of Micro and Nano Science and Technology, Shanghai Jiao Tong University, Shanghai 200030, China.

Color versions of one or more of the figures in this paper are available online at http://ieeexplore.ieee.org.

Digital Object Identifier 10.1109/JMEMS.2008.924274 as infectious diseases, cancers, and inflammatory responses [3]. Unfortunately, extracting information from blood cells can be very challenging due the massive numbers of erythrocytes and the diversity and rareness of the target cells, e.g., a subtype of leukocytes such as $\mathrm{CD} 4^{+} \mathrm{T}$ helper cells, circulating tumor cells, or stem cells. In this paper, we achieved a step toward on-chip blood sample preparation by demonstrating separation of erythrocytes and leukocytes (which contain the target cells for most of the cases) in a simple microfluidic device with a very small footprint, which can be expected to be integrated as a component for on-chip blood analysis.

Conventional methods for cell separation take advantage of different cell properties [4]. For example, centrifugation and gradient centrifugation are widely used for macroscale cell separation and based on differences in buoyant gravity of particles. Immunological techniques, such as fluorescencebased flow cytometry (FACS), magnetic cell sorting (MACS), and affinity chromatography, have been popular, too, because of their specificity and applicability.

Recently, there have been efforts on conducting cell separation in microscale. Many of them have active components which take the advantages of external fields for separation. For examples, field-flow fractionations combine the parabolic flow profile in microchannels with an external field, such as sedimentation, hydrodynamic flow, thermal, and electrical, to separate particles [5]-[7]. Dielectrophoretic (DEP) mechanisms [8], [9] have been used for cell separation either by capturing certain types of cells on an electrode surface with positive DEP force [10] or deflecting certain types of cells from the electrode surface with negative DEP force [11]. Magnetic separators have exploited the weak paramagnetic or diamagnetic difference between erythrocytes and leukocytes in blood to separate them [12]-[14]. More generally, antibody conjugated magnetic particles have been used, as in MACS, to separate a specific type of cells from a heterogeneous mixture of cells [15]. Acoustic standing waves have also been used to continuously separate beads from solution and lipid particles from erythrocytes [16], [17]. Microflow cytometry with fluorescent detection is a general method for biological cell separation, as in traditional FACS. Improvements from traditional FACS are made by replacing glass capillary-based flow chambers with microchannels, integrating compact optics, and providing onchip sample transport [18]-[21].

The other general category for cell separation in microscale is passive devices. They are generally operated by flowing particle suspensions through the device using pressure sources or syringe pumps and, at the same time, separating the particles. There have been several examples for cell separation in 
TABLE I

Comparison of Human Erythrocytes, Leukocytes, and Platelets [44]-[47]

\begin{tabular}{l|c|c|c|c|c}
\hline \multicolumn{1}{c|}{ Cell Type } & Cell Shape & $\begin{array}{c}\text { Diameter } \\
\text { Average }(\boldsymbol{\mu m})\end{array}$ & $\begin{array}{c}\text { Diameter } \\
\text { Range }(\boldsymbol{\mu m})\end{array}$ & $\begin{array}{c}\text { Height } \\
(\boldsymbol{\mu m})\end{array}$ & $\begin{array}{c}\text { Count } \\
(\mathbf{1 0} / \mathbf{L})\end{array}$ \\
\hline Erythrocytes & biconcave disk & 8 & $7-9$ & $1.5-3.0$ & $3.8-5.9 \times 10^{3}$ \\
\hline Leukocytes & sphere & 13 & $6-20$ & $6-20$ & $4.5-11.0$ \\
\hline Platelets & irregular & 3 & $2-4$ & $2-4$ & $1.5-3 \times 10^{2}$ \\
\hline
\end{tabular}

microfabricated devices using mechanical forces. Leukocytes have been separated from whole blood in microchips containing dense arrays of posts spaced at 5 and $7 \mu \mathrm{m}$ and a series of weir-type structures of 3.5- $\mu \mathrm{m}$ height with a capture efficiency between $7 \%$ and $35 \%$ [22]. A series of massively parallel microfabricated sieving device has been used to separate cultured tumor cells from blood in $10 \mu \mathrm{m}$ wide by $20 \mu \mathrm{m}$ deep channels, whereas in a derivative device, leukocytes are separated from erythrocytes in $2.5 \mu \mathrm{m}$ wide by $5 \mu \mathrm{m}$ deep channels [23]. A microstep device has been demonstrated to separate submicrometer polystyrene beads and binary mixtures of bacteria and yeast cells in a shear-driven flow with stepwise tapered microchannel structure [24]. Biomimetic techniques have also been used for cell separation. Granulocytes, lymphocytes, and erythrocytes have been separated due to interaction with polar urethanecoated channel walls and the proposed heteroavoidance between granulocytes and lymphocytes in a lattice of channels of $5 \mu \mathrm{m}$ by $5 \mu \mathrm{m}$ dimension to mimic capillaries [25]. Leukocyteto-erythrocyte ratio has been enriched by 34 -fold in whole blood sample by taking advantage of the intrinsic features of blood flow in the microcirculation, such as plasma skimming and leukocyte margination, in a microfluidic device [26]. A microarray structure coated with E-selectin IgG chimera has been shown to efficiently capture and arrest HL-60 and U-937 cells [27].

More relevant to this paper are examples of passive devices to separate cells hydrodynamically. Bump array devices composed of row-shifted poststructures in a fluidic chamber have demonstrated continuous separation of submicrometer size particles, including large DNA molecules [28], erythrocytes, leukocytes, and plasma [29]-[32], based on deterministic displacement principle. Particle separation in microfluidic bifurcations has been studied [33], [34]. Blood plasma has been separated from blood cells continuously in microchannel bend structures [35], [36] and bifurcating channel structure [37], [38]. The group of M. Seki designs pinch-flow devices [39], [40] and devices with multiple side bifurcated channels [41], [42] and demonstrates continuous separation of beads with different diameters by controlling the fluidic resistance of the branch channels. Recently, the multiple side bifurcated channel device has been demonstrated to separate rat liver cells by size [43]. The underlying principle for these hydrodynamic separators is the same. The particles of micrometer size approximately follow streamlines in the microdevices due to the laminar flow (Reynolds number $R_{e} \ll 1$ ) and the insignificance of the particle diffusion (Peclet number $P_{e} \gg 1$ ). The fluidic streamlines and the geometrical restrictions from the device wall determine the trace of a particle, and critical particle radii for separation can thus be defined. Hydrodynamic separation is attractive because it does not involve moving parts and require active manipulation of particles by other external energy sources. This paper aims specifically to develop a reliable, reusable, continuous, and efficient separation method by using microfluidics for erythrocytes and leukocytes separation.

Human blood cells can be roughly classified into three groups: erythrocytes [red blood cells (RBCs)], leukocytes [white blood cells (WBCs)], and platelets. Leukocytes can be further classified into granulocytes, monocytes, and lymphocytes. Table I compares the geometries and counts of erythrocytes and leukocytes in adults. Generally speaking, leukocytes are larger than erythrocytes. However, there is a wide size range for leukocytes, and the diameters of small lymphocytes overlap with those of erythrocytes. On the other hand, because of its biconcave disk shape, the height of a normal erythrocyte varies from 1.5 to $3.0 \mu \mathrm{m}$, which is clearly smaller than the average leukocyte whose diameter falls between 6 and $20 \mu \mathrm{m}$.

\section{PRINCIPLE}

The principle of the device is based on streamline engineering in the separation region. For the dimension of the device and the flow rate used in testing, the Reynolds number is on the order of $10^{-2}$. The flow inside the devices falls into low Reynolds number laminar flow regime. The center of the particles can be assumed to follow the streamlines of the fluidic field, assuming there are no interactions between the particle and the channel wall and the particle concentration is low enough that particle-particle interaction can be ignored. The separation principle of the device can be illustrated with a T-shape channel in which a side channel branches out at $90^{\circ}$ from the main channel (Fig. 1, top). In Fig. 1, at the bottom, the bifurcated region of the T-shape channel is illustrated with a 2-D fluidic simulation of undisturbed streamlines superimposed. The flow is from right to left and top to bottom. A stagnation point can be identified, which is normally very close to the downstream vertex of the device wall. At this point, the flow has zero velocity. From the stagnation point, a division line can be drawn, which separates the fluid region into two parts. The region between the division line and the device walls, including the side channel, defines the collection zone. If the center of a particle always stays in the collection zone of the main channel, it will be "collected" into the side channel. The vertical distance between the division line and the upstream vertex of the side channel defines the critical particle radius $R$ for separation. The 


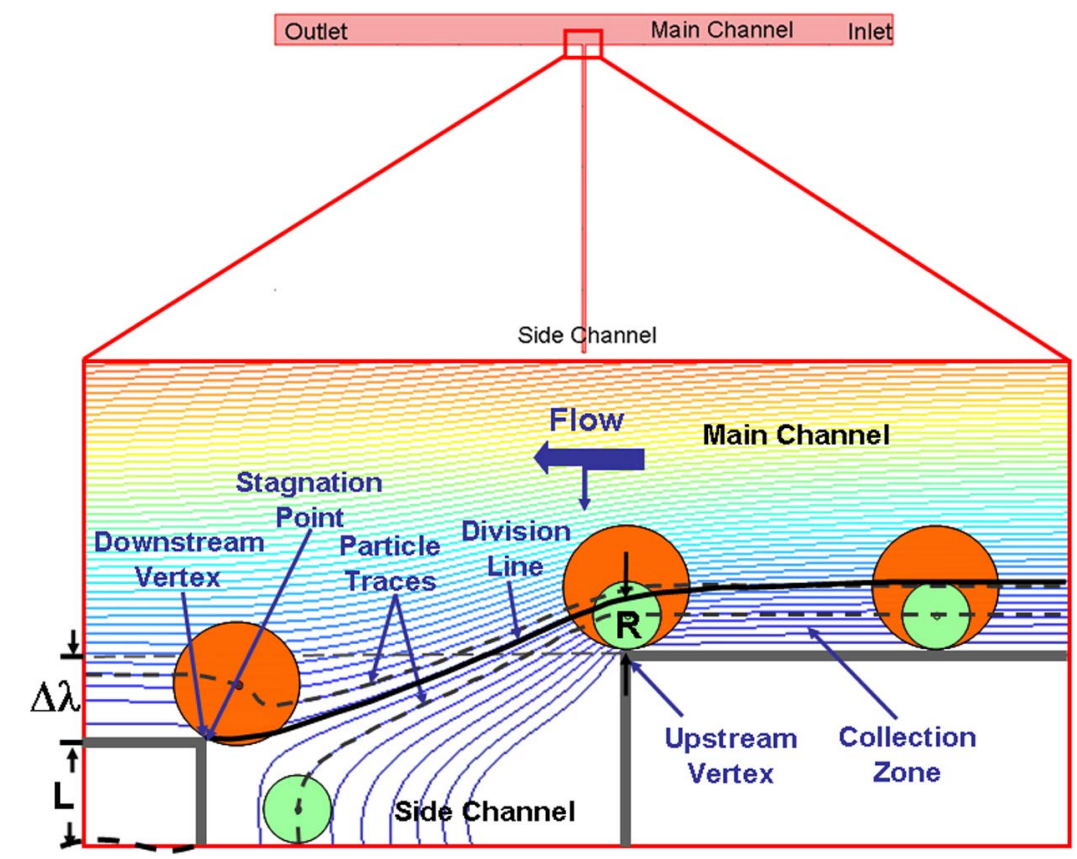

Fig. 1. Principle of separation. Top drawing shows the T-shape channel with bifurcated region labeled by a box. Bottom drawing is a detailed illustration of the boxed area. Background is a 2-D fluidic field simulation with color-coded streamlines. Stagnation point, division line, and collection zone are labeled. Also labeled is the upstream vertex, where the critical particle radius for separation $R$ is defined. The length of side channel $L$ and the vertical distance between downstream and upstream vertices of side channel walls $\Delta \lambda$ are shown. Traces of two particles with radius (orange color) larger and (green color) smaller than $R$ are also illustrated with black dashed lines.

critical particle radius determines the upper limit of a particle that can go into the side channel.

When particles flow into the bifurcated region of the T-shape channel, they are forced to flow along the bottom channel wall, as shown in Fig. 1, to ensure that the particles are subjected to the same separation conditions. This can be accomplished by using pinched flow, which will be shown later in Fig. 3. When there is no interference from the wall, the particle will flow along the same streamline. The particle-device wall interaction might shift the center of a particle to a new streamline. For a particle whose radius is smaller than the critical particle radius $R$, its center always stays inside the collection zone; thus, it flows into the side channel. For a particle whose radius is larger than $R$, its center is above the division line at the upstream vertex and, hence, never enters the collection zone afterward.

\section{SimULATION}

The separation is determined by the critical particle radius $R$. We studied two factors that can be used to control $R$ : the length of side channel and the vertical distance between downstream and upstream side channel vertices. The T-shape channel was served as our model. The effects were studied by the 2-D finite element simulation of fluidic field with the Navier-Stokes equation (Comsol Multiphysics, Comsol, Inc.). An aqueous solution was assumed to be flowing inside the device. Parabolic velocity profile with maximal velocity of $1 \mathrm{~mm} / \mathrm{s}$ was applied at the inlet. Zero pressure was used for outlets. The no-slip condition was assumed at the device walls. If not specified otherwise, $1000 \mu \mathrm{m}$ was used as channel length for inlet, main outlet, and side outlet channels. $100 \mu \mathrm{m}$ was used as channel width for both inlet and main outlet channels, whereas $10 \mu \mathrm{m}$ was used as the channel width of the side channel. Hydrodynamic streamlines in the T-shape channel were generated by simulation, and the corresponding critical particle radius was measured from the simulation directly, according to the definition in Fig. 1.

First, we examine the effect of the length of side channel $L$. As the solid line generated with finite element simulation in Fig. 2(a) shows, increasing side channel length decreases critical particle radius. This is caused by larger fluidic resistance in the side channel and, hence, a reduced flow rate in the side channel and can be approximated analytically. For low Reynolds number flow in a long tube, pressure difference is proportional to flow rate. The ratio of pressure difference and flow rate defines fluidic resistance. In 2-D, fluidic resistance can be expressed as

$$
R=\frac{12 \mu L}{W^{3}}
$$

where $W$ is channel width, $L$ is channel length, and $\mu$ is fluid viscosity. Applying the flow resistance model to the T-shape channel, the main inlet, main outlet, and side channel outlet are simulated as three fluidic resistors. The fluidic resistance of the main outlet is in parallel with that of the side channel. From the flow rate ratio of the main outlet and the side channel and assuming a parabolic flow profile of velocity field in the main inlet, the width of fluid in the main inlet channel that goes into side channel is calculated. The result is shown in Fig. 2(a) as the dashed line. The values calculated with the fluidic resistance model are always larger than those obtained by simulation. This discrepancy is caused by the fact that the parabolic velocity profile can only be assumed far upstream from the upstream vertex, whereas the critical particle radius is 

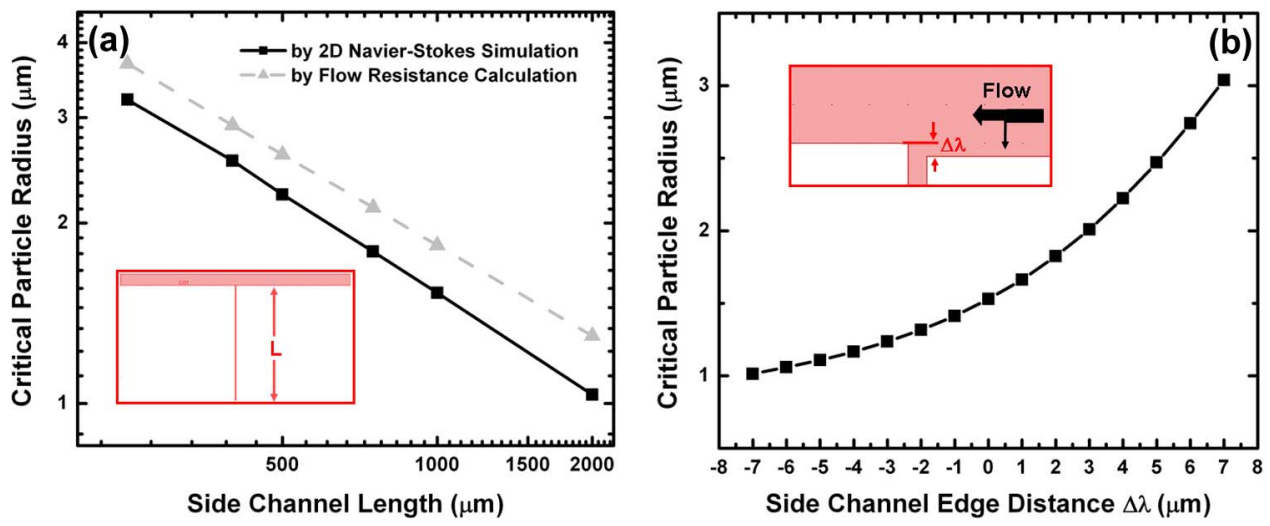

Fig. 2. Simulation study on critical particle radius $R$. (a) Effect of side channel length $L$. Solid line represents the results of 2-D Navier-Stokes simulation. Dashed line was the calculation from the flow resistance model. (b) Effect of side channel edge distance $\Delta \lambda$. Positive $\Delta \lambda$ was defined, as inset.

defined right at the upstream vertex. It is confirmed in our finite element simulation results, where we find that the calculated critical particle radius by the fluidic resistance model matches exactly with the distance between the division line and the main channel wall at far upstream of the upstream vertex.

The other factor we consider is the local geometry of the bifurcated region. In this case, the vertical distance between downstream and upstream side channel vertices $\Delta \lambda$, as shown in Fig. 1, is used as a design parameter. As in Fig. 2(b), generated from finite element simulation, critical particle radius $R$ increases as $\Delta \lambda$ increases, and it is more sensitive when $\Delta \lambda$ is positive. $\Delta \lambda$ is a local property, and it is not considered by the fluidic resistance model. The simulation results in this paper show that it is a parameter that can significantly change the critical particle radius. Compared with the side channel length, a design with local geometry may reduce chip area by avoiding very long side channels.

\section{EXPERIMENTAL}

\section{A. Material}

Polydimethylsiloxane [(PDMS); Sylgard 184] was obtained from Dow Corning, Midland, MI. Polystyrene beads were purchased from Duke Scientific Corporations, Fremont, CA. Beads with $5-\mu \mathrm{m}$ diameter are dyed with red fluorophore, whereas $10-\mu$ m-diameter ones are dyed with green fluorophore. The beads are diluted to $10^{5} / \mathrm{mL}$ range before use. Wintrobe tubes of $115 \mathrm{~mm}$ long and 3-mm inner diameter were purchased from Chase Scientific (R828B, Rockwood, TN). Fresh human blood was collected from healthy donors in anticoagulant-EDTAadded collection tubes. Blood samples were used within $48 \mathrm{~h}$ of withdrawal. Leukocyte rich plasma (LRP) was prepared with Wintrobe tubes as follows. One milliliter of bubble-free fresh whole blood was filled into the tube. The tube was laid on flat surface for $30 \mathrm{~min}$ to an hour. Then, it was gradually tilted up to vertical position. The upper clear plasma part was carefully transferred to a clean container.

Human blood cell separation was demonstrated by using diluted whole blood for erythrocyte characterization and LRP for leukocyte measurements. In both cases, the blood was diluted with Ficoll-Paque Plus (Amersham Biosciences, Sweden). The major component of Ficoll-Paque Plus is Ficoll 400, which is a neutral highly branched hydrophilic polymer of sucrose. In tradition, it has been used as a reagent to purify lymphocytes from human peripheral blood based on density gradient centrifugation. Its density is $1.077 \pm 0.001 \mathrm{~g} / \mathrm{mL}$, which is closer to blood cell density than aqueous saline. Diluting blood with Ficoll-Paque Plus keeps blood cells alive and enables us to run experiment over $1 \mathrm{~h}$ without severe sedimentation. Leukocytes in LRP were stained with fluorescent cell nucleus dye acridine orange (Molecular Probes, Eugene, OR) at a final concentration of $10 \mu \mathrm{g} / \mathrm{mL}$. Because erythrocytes do not contain nuclei, only leukocytes are stained by acridine orange.

\section{B. Device Design}

We designed and tested two devices. For device I, we wanted to test only the effect of local geometry changes. We kept side channel length $L$ constant and only changed the vertical distance $\Delta \lambda$. To optimize for blood cell separation, we changed both $L$ and $\Delta \lambda$ in device II to achieve a wider range of critical particle radii.

Both devices contained one sample inlet, one buffer inlet, one main outlet, and multiple side channel outlets (Fig. 3). Three side channels with the same local geometry were grouped into one at downstream. There are 11 of these side channel groups in device I and ten in device II. After a sample flow met a buffer flow, the sample flow was pushed against the device wall by the buffer flow in the pinch-flow region. Relative flow rates from buffer flow to sample flow were adjusted to a ratio of about ten, so that all particles in the sample flow aligned against the channel wall of the sample flow side before they entered the separation region. The pinch-flow region was $500 \mu \mathrm{m}$ long, so that the flow was stabilized before it entered the separation region. The separation region consisted of an array of bifurcated channels that are perpendicular to the main channel, each with a corresponding critical particle radius.

The critical particle radius for each side channel group was calculated from the finite element simulation of the corresponding T-channels. Simulation conditions are similar to the conditions used in the experimental part. Compared with calculating all the critical particle radii simultaneously from 


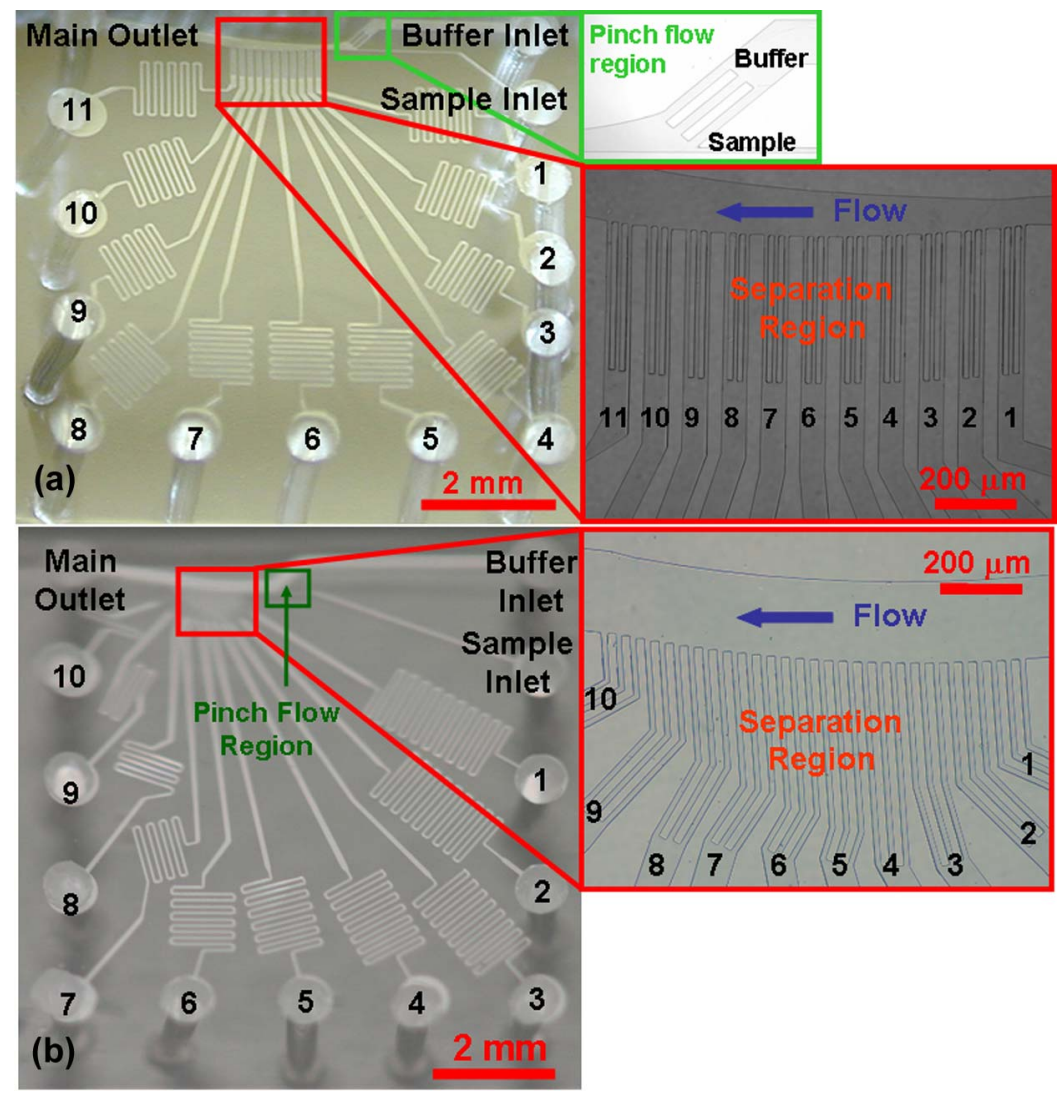

Fig. 3. Design of devices. (a) Device I with only $\Delta \lambda$ changed for side channels. (b) Device II with both $L$ and $\Delta \lambda$ changed for side channels.
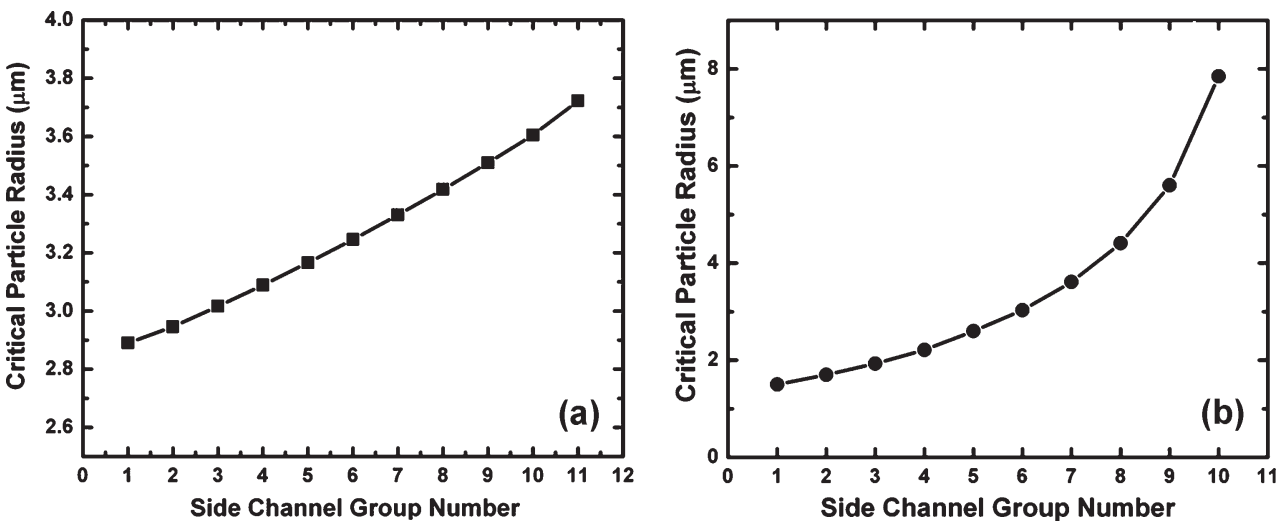

Fig. 4. Prediction of critical particle radii $R$ for different side channel groups in (a) device I and (b) device II.

the whole chip simulation, the simulation of T-channels can be performed easily on a PC with adequate precision. Moreover, it is a preferred design approach by being able to consider one single side channel at once without being worried about the coupling effects from other side channels at the early design stage and then integrate all the side channels together later without changing the design parameters. This approach is valid due to the configuration of the devices. The main inlet (combination of the sample inlet and the buffer inlet) and the main outlet are much wider and shorter than the side channels. Therefore, their flow resistances are much smaller. Moreover, the bifurcation points of the side channels are very close. In consequence, the ratio of pressure at the bifurcation points to the overall pressure is determined and fixed by the flow resistances of the main inlet and the main outlet, and adding side channels does not significantly change this ratio. This is the reason that the T-shape channel simulation can be used to simulate individual corresponding side channel in a complicated device.

In device $\mathrm{I}, \Delta \lambda$ increased from -5 to $5 \mu \mathrm{m}$ in a $1-\mu \mathrm{m}$ increment from upstream to downstream for the 11 groups of side bifurcated channels. Simulation with T-shape channels predicted the critical particle radius for each of the side channel group, as shown in Fig. 4(a). From side channel group number 1 to 11 , the critical particle radius increases from 2.9 to $3.8 \mu \mathrm{m}$.

To optimize the device for blood cell separation, a device with a larger span of critical particle radius is desirable. Erythrocytes average $8 \mu \mathrm{m}$ in diameter and can range from 7 to $9 \mu \mathrm{m}$ (Table I). Leukocytes have a wider range, which is 

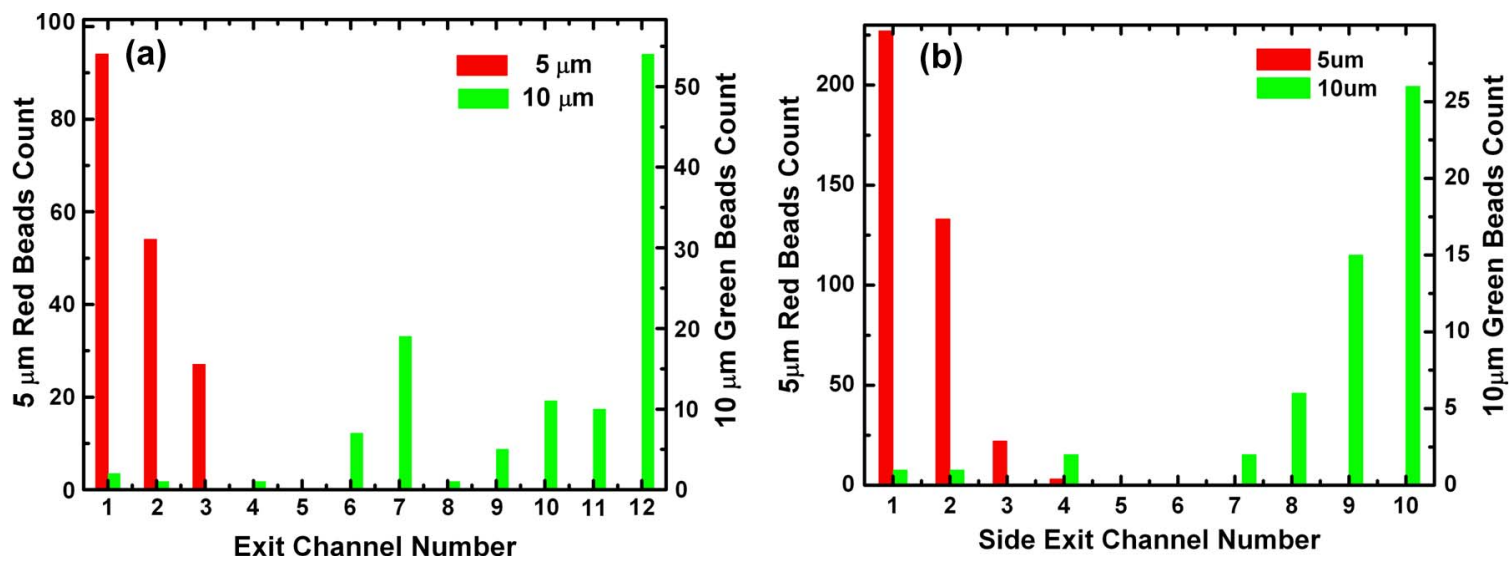

Fig. 5. Statistics of separation of 5- and 10- $\mu$ m polystyrene beads. (a) Device I: Side exit channels numbered 1 to 11 are side bifurcated channels, and side exit channel 12 is the main outlet. (b) Device II: Side exit channels numbered 1 to 10 are all side bifurcated channels.

from 6 to $20 \mu \mathrm{m}$. The ratio of erythrocytes to leukocytes in whole human blood is in the magnitude of thousands to one. For device II, from upstream to downstream, $\Delta \lambda$ increases from 0 to $9 \mu \mathrm{m}$ in a $1-\mu \mathrm{m}$ increment, and the lengths of the exit channels decrease linearly from 22000 to $2200 \mu \mathrm{m}$ in a $-2200-\mu \mathrm{m}$ increment. The critical particle radii predicted by finite element simulation are shown in Fig. 4(b). From side channel group one to ten, the critical particle radius increases roughly exponentially in a range from 1.50 to $7.85 \mu \mathrm{m}$. This is desirable, because the majority of cells in blood fall into the lower size range. With this design, the separation is finer for smaller particles and rougher for large ones.

\section{Device Fabrication}

We chose PDMS as the device material because of its blood compatibility and ease of use. The mold for PDMS was fabricated by deep reactive ion etching on a silicon wafer with photoresist AZ4620 as a mask. The channel height for device I is $40 \mu \mathrm{m}$, and that of device II is $20 \mu \mathrm{m} .1,1,1,3,3$, 3-hexamethyldisilazane was used to treat the mold before applying homogenous mixture of PDMS parts A and B (10:1). The PDMS was cured with mold at $80{ }^{\circ} \mathrm{C}$ for $25 \mathrm{~min}$. Then, it was separated from the mold, cut into pieces, and punched with Luer stub adapter (gauge 20, Becton Dickinson, NJ) for access holes. PDMS pieces were then ultrasonically cleaned in acetone for $10 \mathrm{~min}$ and rinsed with acetone and then isopropyl alcohol (IPA). Finally, PDMS pieces were mounted on clean glass slides by overnight baking at $80{ }^{\circ} \mathrm{C}$.

\section{Device Testing}

The operation of devices I and II was first validated with fluorescent polystyrene beads of 5 and $10 \mu \mathrm{m}$ in diameter. Human blood cell separation was demonstrated in device II. Sample flow rate was kept at $0.06 \mu \mathrm{L} / \mathrm{min}$, whereas buffer flow was in the range of $0.5-0.8 \mu \mathrm{L} / \mathrm{min}$. The interface of the sample flow and buffer flow could be clearly observed under a microscope. To minimize the effect of bead-to-bead interaction, total bead concentration was on the order of $100 \mu \mathrm{L}^{-1}$.
Flows were introduced into the device by syringe pumps (pump 11 Pico Plus, Harvard Apparatus, MA). The separation region of the device was observed under fluorescent microscope (Nikon E800, Japan) during the whole testing period. A chargecoupled device (CCD) camera (Spot RT slider, Diagnostic Instruments, MI) was connected to the microscope and used to take videos at various frame rates due to different levels of illumination. Videos were later analyzed frame by frame.

\section{RESUlts AND Discussion}

The results of the separation of 5 - $\mu$ m-diameter green and $10-\mu$ m-diameter red fluorescent beads in device I are shown in Fig. 5(a). All 5- $\mu \mathrm{m}$ beads exited from the side channel group numbers one to three, with number one capturing the most. Approximately $50 \%$ of the $10-\mu \mathrm{m}$ beads exited from the main outlet, as predicted by our simulation of the critical particle radius; the rest flew into side bifurcated channels. Assuming side channels one to three were collected for $5-\mu \mathrm{m}$ beads and the rest exit channels were collection for $10-\mu \mathrm{m}$ beads, the normalized separation efficiency was $97 \%$ for device I to separate 5- and $10-\mu \mathrm{m}$ beads, meaning that, with inputting of equal number of 5 - and $10-\mu \mathrm{m}$ beads, over $97 \%$ of the cells in exit groups one to three are 5- $\mu \mathrm{m}$ beads and the same for the $10-\mu \mathrm{m}$ beads in exit groups four to ten.

In device II, $5-\mu \mathrm{m}$ beads exited mainly from side channel groups one and two (Fig. 6). The predicted critical particle radii were 1.50 and $1.70 \mu \mathrm{m}$. The $10-\mu \mathrm{m}$ beads exited mainly from side channel groups eight to ten. The predicted critical particle radii ranged from 4.41 to $7.85 \mu \mathrm{m}$, which matched well with the average radius of the particles. If the $5-\mu \mathrm{m}$ beads were assumed to exit from side channel groups one to three and the $10-\mu \mathrm{m}$ beads from groups four to ten, the histogram of separation profile [Fig. 5(b)] showed that the separation had an efficiency of $96 \%$.

There are some discrepancies between predicted critical particle radii and separation profile for large beads of $10-\mu \mathrm{m}$ diameter in device I and small beads of $5-\mu \mathrm{m}$ diameter in device II. In device I, according to simulation, all 10- $\mu$ m beads should exit from the main outlet. However, in testing, about $50 \%$ of the $10-\mu \mathrm{m}$ beads exited from side channel groups 


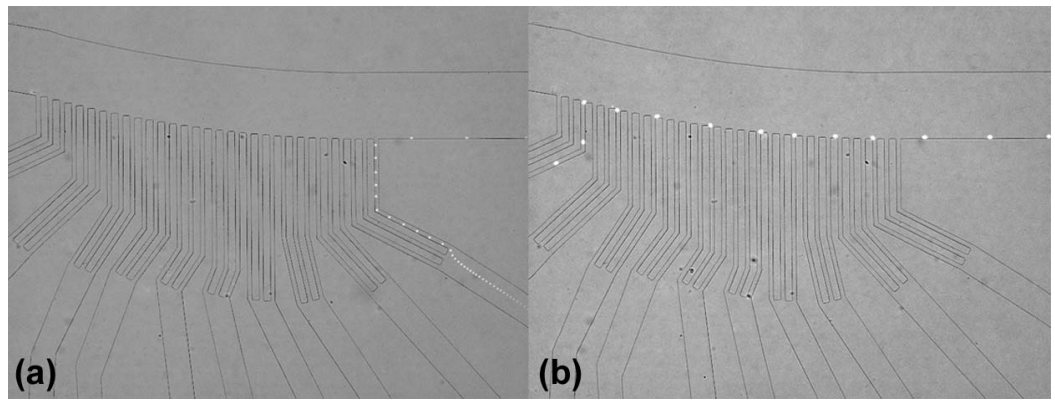

Fig. 6. Typical traces of (a) a 5- $\mu \mathrm{m}$ fluorescent polystyrene bead and (b) a 10- $\mu \mathrm{m}$ fluorescent polystyrene bead in device II.
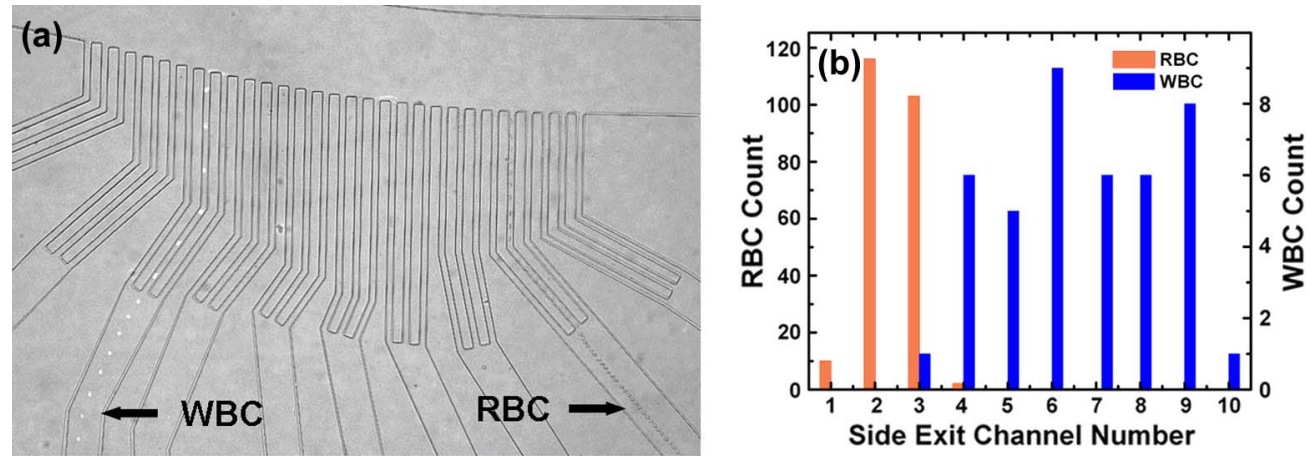

Fig. 7. Erythrocytes and leukocytes separation in device II. (a) Typical traces of erythrocyte (RBC) and leukocyte (WBC) in device II, as pointed by arrows. For the RBC case, images were extracted from a video with bright field illumination and then overlaid. The images of WBC were extracted from a video with both weak bright field and Epi fluorescent illumination and then overlaid. (b) Separation statistics of erythrocytes and leukocytes in LRP.

6 to 11. In device II, the simulation predicted most of the 5 - $\mu$ m-diameter particles would exit from side channel groups four and five. However, in testing, they mainly exited from side channel groups one and two. These could be due to the coupling effect of side bifurcated channels, which was not considered in T-shape simulation, and/or the size variation of the beads (up to $15 \%$ ). Other factors, such as the unsteady flow fluctuation caused by the syringe pumps, adhesion of particles to device wall, and the difference between 2-D fluidic simulation and 3-D device operation, could contribute to the discrepancies. In general, device II demonstrated better separation profile for $10-\mu \mathrm{m}$ particles. In that device, the step sizes of critical particle radii for larger particles were larger.

For both devices, we observed under a microscope that the $10-\mu \mathrm{m}$ beads flow in a "jumping" mode in the separation region, meaning they move toward the stagnation points close to the downstream vertexes and slow down. After passing these stagnation points, they quickly flow toward the next side bifurcated channel. This is similar to the displace mode flow of large particles in the bump array devices [28].

Erythrocyte and leukocyte separation using diluted human blood was performed with device II. Erythrocyte behavior inside the device was studied by using whole human blood with 1000 times dilution. It still had erythrocyte to leukocyte ratio in the range of a thousand. The cell flow inside the device was recorded with a digital camera in video recording mode under a bright field illumination of a microscope. Erythrocytes exit mainly from side channel groups two and three. A typical erythrocyte trace was obtained by overlying images of an erythrocyte flowing inside the device [Fig. 7(a)]. On the same picture shows a typical leukocyte trace inside the device obtained in an experiment described hereafter. To study leukocytes flow inside the device, LRP was prepared, stained with acridine orange, and tested with a final concentration of leukocytes in the range of $10^{2} \mu \mathrm{L}^{-1}$. The ratio of erythrocytes to leukocytes was in tens to hundreds range. The cell flow inside the device was recorded with a digital camera in video recording mode under green Epi fluorescent and weak bright field illumination of a fluorescent microscope. Cell separation statistics was obtained from the analysis of the video [Fig. 7(b)]. The leukocytes are bright green fluorescent due to the acridine orange staining. The erythrocytes are dark under the weak bright field illumination, and they can be analyzed from the video in slow play modes. The leukocytes demonstrated a different separation profile from erythrocytes. Most of the leukocytes exited from side channel groups four to nine, whereas erythrocytes still exited mainly from side channel groups two and three. Not shown in Fig. 7(b), we also observed one leukocyte exiting from the main outlet during testing. If a line was drawn between exit groups three and four with the assumption that erythrocytes exited from group numbers one to three and leukocytes exited from the rest, a separation efficiency of $97 \%$ was achieved. The portion of the separation profile that erythrocytes and leukocytes did not separate very well was likely to be caused by the size overlap between erythrocytes and small lymphocytes. Although the volume of average small lymphocytes is at least twice that of erythrocytes [48], [49], the size is the determinant factor for the separation inside this device. The diameters of erythrocytes are reported between 7 and $9 \mu \mathrm{m}$, whereas those of small lymphocytes are between 6 and $9 \mu \mathrm{m}$ [44], [46], [47]. 
On the other hand, because of the biconcave disk shape of erythrocytes, their heights range from 1.5 to $3.0 \mu \mathrm{m}$, which are substantially smaller than the average diameter of small lymphocytes. Therefore, the separation of erythrocytes and small lymphocytes inside the device depended heavily on the orientation of erythrocytes in the separation region. In our testing, we observed a large percentage of erythrocytes with the flat portion of the disk shape aligned to the device wall before they entered the separation region, which could help the separation performance. It will be beneficial to increase the flow rates so that the shear stress of fluid can better align the erythrocytes along the device wall and the comparison of their heights (instead of their diameters) to critical particle radius will determine into which side channel they flow.

There are several benefits offered by the devices. The filtering effect of the device was achieved by the collection zone formed by the fluidic field, not by physical size limitation. Moreover, this filtering effect was in the direction perpendicular to the flow direction. This greatly reduced clogging. The separation regions of the devices had a very small footprint of $1.5 \mathrm{~mm}$ by $0.8 \mathrm{~mm}$. Setting up fluidic resistance of the side channels could take up a lot of chip space. We showed in this paper that the local geometry design of the separation region $(\Delta \lambda)$ could change the critical particle radius, which could reduce the required length of the side channels. In our designs, the length and width of the side channels are not optimized for saving chip space, but rather as a convenient way to spread out for outlet connections. By reducing side channel width and length simultaneously, the chip area of the side channels can be reduced by about two orders of magnitude with the same performance for blood cell separation. This makes the device very desirable as a component for blood sample preparation and analysis. Particles of different sizes were collected in different reservoirs that are connected to the side channels. This could facilitate further characterization of the particles. The solvent of sample flow went into upstream side channels. This meant that larger particles, which exited from downstream side channels or main outlet, flowed out with the solvent in the buffer flow, which made solvent change for larger particles possible.

Flow control could be a problem for the operation of the device. Although unlike a lot of previous passive particle separators, hydrodynamic focusing is not required at the inlet, stable fluidic fields are still required. Disturbance from pumps, particle adhesion to device walls, and particle-fluid and particle-particle interactions could change the local flow and thus change the separation profile and efficiency. A multiple port injector structure [top right inset in Fig. 3(a)] was designed in the buffer flow inlet. As a result, although the buffer flow channel was wide, the flow was stable and laminar [50]. Particle-particle interactions set an upper limit on the concentration of the particles. The highest blood concentration we tried with good separation profile is on the order of 100 times diluted whole blood. Particle adhesion to device wall could degrade device performance, particularly in testing with LRP. Platelets and blood cells were observed to adhere to the device wall gradually, and plasma protein could build up on device surface. This process is related to the freshness of the blood sample. For fresh blood withdrawn within $24 \mathrm{~h}$, it took more than $1 \mathrm{~h}$ before significant biomass built up inside the device under our testing conditions. For aged blood samples that are older than three days, the effect showed up within half an hour of device operation. Surface treatment and dynamic coating could improve the situation by preventing cell adhesion and protein buildup on the device wall.

The experimental results presented in this paper use low flow rates and high particle dilution for the convenience of data recording and analysis. We also achieved similar separation results by increasing the flow rates to $1 \mu \mathrm{L} / \mathrm{min}$ and reducing blood dilution to 100 times. The throughput of the system can reach $\sim 10^{3}$ cells per second in this case, which is comparable to typical desktop FACS systems.

Two-dimensional Navier-Stokes fluidic dynamic simulation was used for critical particle radius prediction in device design. For streamline design with local geometry as in the case of device I, fluidic resistance model could not be applied, and simulation was required. In Fig. 2(a), even in cases where only lengths of side channels change and, thus, fluidic resistance model was applicable, using simulation was more accurate than using fluidic resistance model for critical particle radius prediction. This is because the critical particle radius is a property determined by local streamlines at the bifurcating region, which is closely related to the local geometrical design. Unfortunately, this cannot be considered in the fluidic resistance model. For devices with local geometry variations $(\Delta \lambda)$, streamline-based design with simulation was used to predict the critical particle radius. Although the testing results showed a good match between 2-D fluidic simulation and testing results, the 3-D nature of the channels may have some effects on particle separation. Ideally for very deep channels, the parabolic velocity profile of the fluid and the resultant shear stress will keep particles of similar size in the same layer of fluid. This can make the flow effectively 2-D. Making high aspect ratio channels could be a problem in device fabrication; in our PDMS on glass devices, the aspect ratio was under two.

Assuming that a particle follows the same streamline in low Reynolds number, laminar flow is an approximation. The actual particle trajectory is more complicated. The flow shears on the particles, the rotation of particles, and the nonspherical shapes of particles contribute to the transverse lift acting on particles [51]. In the case of blood flow in capillary, blood cells have been reported to be kept away from capillary walls [52]. Further investigation of these theoretical aspects can benefit the design of future passive hydrodynamic microseparators.

\section{CONCLUSION}

We presented a streamline-based design of microfabricated devices to achieve particle separation by size. The 2-D NavierStokes simulation was used to predict the critical particle radius for separation, which was the parameter that determines the binary nature of the separation. Two devices were designed and fabricated. Both of them separated 5- and $10 \mu \mathrm{m}$-diameter polystyrene beads efficiently. The second device also demonstrated effective separation of human erythrocytes and leukocytes. It had a wider range of critical particle radii with finer 
separation for small particles. This made it better suited for human blood cell separation.

\section{ACKNOWLEDGMENT}

The authors would like to thank all of the members of the Caltech Micromachining Group for their valuable assistance.

\section{REFERENCES}

[1] J. El-Ali, P. K. Sorger, and K. F. Jensen, “Cells on chips," Nature, vol. 442, no. 7101, pp. 403-411, Jul. 27, 2006.

[2] H. Andersson and A. van den Berg, "Microfluidic devices for cellomics: A review," Sens. Actuators B, Chem., vol. 92, no. 3, pp. 315-325, Jul. 15, 2003.

[3] M. Toner and D. Irimia, "Blood-on-a-chip," Annu. Rev. Biomed. Eng., vol. 7, no. 1, pp. 77-103, Aug. 2005.

[4] D. Recktenwald and A. Radbruch, Cell Separation Methods and Applications. New York: Marcel Dekker, 1998.

[5] J. C. Giddings, "Field-flow fractionation: Analysis of macromolecular, colloidal, and particulate materials," Science, vol. 260, no. 5113, pp. 1456-1465, Jun. 4, 1993.

[6] Y. Jiang, M. N. Myers, and J. C. Giddings, "Separation behavior of blood cells in sedimentation field-flow fractionation," J. Liq. Chromatogr. Relat. Technol., vol. 22, no. 8, pp. 1213-1234, 1999.

[7] J. Yang, Y. Huang, X. B. Wang, F. F. Becker, and P. R. C. Gascoyne, "Cell separation on microfabricated electrodes using dielectrophoretic/ gravitational field-flow fractionation," Anal. Chem., vol. 71, no. 5, pp. 911-918, Mar. 1, 1999.

[8] M. P. Hughes, "Strategies for dielectrophoretic separation in laboratoryon-a-chip systems," Electrophoresis, vol. 23, no. 16, pp. 2569-2582, Aug. 2002.

[9] P. R. C. Gascoyne and J. V. Vykoukal, "Dielectrophoresis-based sample handling in general-purpose programmable diagnostic instruments," Proc. IEEE, vol. 92, no. 1, pp. 22-42, Jan. 2004.

[10] F. F. Becker, X. B. Wang, Y. Huang, R. Pethig, J. Vykoukal, and P. R. C. Gascoyne, "Separation of human breast cancer cells from blood by differential dielectric affinity," Proc. Nat. Acad. Sci. USA, vol. 92, no. 3, pp. 860-864, Jan. 31, 1995

[11] X. Hu, P. H. Bessette, J. Qian, C. D. Meinhart, P. S. Daugherty, and H. T. Soh, "Marker-specific sorting of rare cells using dielectrophoresis," Proc. Nat. Acad. Sci. USA, vol. 102, no. 44, pp. 15757-15761, Nov. 1, 2005.

[12] K.-H. Han and A. B. Frazier, "Continuous magnetophoretic separation of blood cells in microdevice format," J. Appl. Phys., vol. 96, no. 10, pp. 5797-5802, Nov. 15, 2004.

[13] K.-H. Han and A. B. Frazier, "Diamagnetic capture mode magnetophoretic microseparator for blood cells," J. Microelectromech. Syst., vol. 14 , no. 6, pp. 1422-1431, Dec. 2005.

[14] K.-H. Han and A. B. Frazier, "Paramagnetic capture mode magnetophoretic microseparator for high efficiency blood cell separations," Lab Chip, vol. 6, no. 2, pp. 265-273, Feb. 2006.

[15] D. W. Inglis, R. Riehn, J. C. Sturm, and R. H. Austin, "Microfluidic high gradient magnetic cell separation," J. Appl. Phys., vol. 99, no. 8, pp. 08K 101-1-08K 101-3, Apr. 15, 2006.

[16] A. Nilsson, F. Petersson, H. Jonsson, and T. Laurell, "Acoustic control of suspended particles in micro fluidic chips," Lab Chip, vol. 4, no. 2, pp. 131-135, Apr. 2004.

[17] F. Petersson, A. Nilsson, C. Holm, H. Jonsson, and T. Laurell, "Continuous separation of lipid particles from erythrocytes by means of laminar flow and acoustic standing wave forces," Lab Chip, vol. 5, no. 1, pp. 2022, Jan. 2005.

[18] D. Huh, W. Gu, Y. Kamotani, J. B. Grotberg, and S. Takayama, "Microfluidics for flow cytometric analysis of cells and particles," Physiol. Meas., vol. 26, no. 3, pp. R73-R98, Jun. 2005.

[19] A. Y. Fu, C. Spence, A. Scherer, F. H. Arnold, and S. R. Quake, "A microfabricated fluorescence-activated cell sorter," Nat. Biotechnol., vol. 17, no. 11, pp. 1109-1111, Nov. 1999.

[20] L.-M. Fu, R.-J. Yang, C.-H. Lin, Y.-J. Pan, and G.-B. Lee, "Electrokinetically driven micro flow cytometers with integrated fiber optics for on-line cell/particle detection," Anal. Chim. Acta, vol. 507, no. 1, pp. 163-169, Apr. 1, 2004.

[21] A. Wolff, I. R. Perch-Nielsen, U. D. Larsen, P. Friis, G. Goranovic, C. R. Poulsen, J. P. Kutter, and P. Telleman, "Integrating advanced func- tionality in a microfabricated high-throughput fluorescent-activated cell sorter," Lab Chip, vol. 3, no. 1, pp. 22-27, Feb. 2003.

[22] P. Wilding, L. J. Kricka, J. Cheng, G. Hvichia, M. A. Shoffner, and P. Fortina, "Integrated cell isolation and polymerase chain reaction analysis using silicon microfilter chambers," Anal. Biochem., vol. 257, no. 2, pp. 95-100, Mar. 15, 1998.

[23] H. Mohamed, L. D. McCurdy, D. H. Szarowski, S. Duva, J. N. Turner, and M. Caggana, "Development of a rare cell fractionation device: Application for cancer detection," IEEE Trans. Nanobiosci., vol. 3, no. 4, pp. 251-256, Dec. 2004.

[24] S. Vankrunkelsven, D. Clicq, K. Pappaert, W. Ranson, C. De Tandt, H. Ottevaere, H. Thienpont, G. V. Baron, and G. Desmet, "A novel microstep device for the size separation of cells," Electrophoresis, vol. 25, no. 10/11, pp. 1714-1722, Jun. 2004.

[25] R. H. Carlson, C. V. Gabel, S. S. Chan, R. H. Austin, J. P. Brody, and J. W. Winkelman, "Self-sorting of white blood cells in a lattice," Phys. Rev. Lett., vol. 79, no. 11, pp. 2149-2152, Sep. 15, 1997.

[26] S. S. Shevkoplyas, T. Yoshida, L. L. Munn, and M. W. Bitensky, "Biomimetic autoseparation of leukocytes from whole blood in a microfluidic device," Anal. Chem., vol. 77, no. 3, pp. 933-937, Feb. 2005.

[27] W. C. Chang, L. P. Lee, and D. Liepmann, "Biomimetic technique for adhesion-based collection and separation of cells in a microfluidic channel," Lab Chip, vol. 5, no. 1, pp. 64-73, Jan. 2005.

[28] L. R. Huang, E. C. Cox, R. H. Austin, and J. C. Sturm, "Continuous particle separation through deterministic lateral displacement," Science, vol. 304, no. 5673, pp. 987-990, May 2004.

[29] J. A. Davis, D. W. Inglis, K. J. Morton, D. A. Lawrence, L. R. Huang, S. Y. Chou, J. C. Sturm, and R. H. Austin, "Deterministic hydrodynamics: Taking blood apart," Proc. Nat. Acad. Sci. USA, vol. 103, no. 40, pp. 14779-14784, Oct. 2006.

[30] D. W. Inglis, J. A. Davis, R. H. Austin, and J. C. Sturm, "Critical particle size for fractionation by deterministic lateral displacement," Lab Chip, vol. 6, no. 5, pp. 655-658, May 2006.

[31] S. Zheng, R. Yung, Y.-C. Tai, and H. Kasdan, "Deterministic lateral displacement MEMS device for continuous blood cell separation," in Proc. 18th IEEE Int. Conf. MEMS, Miami Beach, FL, 2005, pp. 851-854.

[32] S. Zheng, Y.-C. Tai, H. Lin, M. Balic, R. Datar, and R. J. Cote, "Circulating tumor cell capture from whole blood by parylene filter devices," in Proc. MicroTAS, Boston, MA, 2005.

[33] B. W. Roberts and W. L. Olbricht, "Flow-induced particulate separations,' AICHE J., vol. 49, pp. 2842-2849, Nov. 2003

[34] B. W. Roberts and W. L. Olbricht, "The distribution of freely suspended particles at microfluidic bifurcations," AICHE J., vol. 52, no. 1, pp. 199206, Jan. 2006

[35] C. Blattert, R. Jurischka, A. Schoth, P. Keith, and W. Menz, "Separation of blood in microchannel bends," in Proc. 25th Annu. Int. Conf. IEEE EMBS, Cancun, Mexico, 2003, pp. 3388-3391.

[36] C. Blattert, R. Jurischka, I. Tahhan, A. Schoth, P. Kerth, and W. Menz, "Microfluidic blood/plasma separation unit based on microchannel bend structures," in Proc. 3rd IEEE/EMBS Spec. Top. Conf. Microtechnol. Med. Biol., Kahuku, HI, 2005, pp. 38-41.

[37] S. Yang, A. Undar, and J. D. Zahn, "A microfluidic device for continuous, real time blood plasma separation," Lab Chip, vol. 6, no. 7, pp. 871-880, Apr. 2006.

[38] R. D. Jaggi, R. Sandoz, and C. S. Effenhauser, "Microfluidic depletion of red blood cells from whole blood in high-aspect-ratio microchannels," Microfluidics Nanofluidics, vol. 3, no. 1, pp. 47-53, Feb. 2007.

[39] J. Takagi, M. Yamada, M. Yasuda, and M. Seki, "Continuous particle separation in a microchannel having asymmetrically arranged multiple branches," Lab Chip, vol. 5, no. 7, pp. 778-784, Jul. 2005.

[40] M. Yamada, M. Nakashima, and M. Seki, "Pinched flow fractionation: Continuous size separation of particles utilizing a laminar flow profile in a pinched microchannel," Anal. Chem., vol. 76, no. 18, pp. 5465-5471, Aug. 2004.

[41] M. Yamada and M. Seki, "Microfluidic particle sorter employing flow splitting and recombining," Anal. Chem., vol. 78, no. 4, pp. 1357-1362, Feb. 15, 2006.

[42] M. Yamada and M. Seki, "Hydrodynamic filtration for on-chip particle concentration and classification utilizing microfluidics," Lab Chip, vol. 5, no. 11, pp. 1233-1239, Nov. 2005.

[43] M. Yamada, K. Kano, Y. Tsuda, J. Kobayashi, M. Yamato, M. Seki, and T. Okano, "Microfluidic devices for size-dependent separation of liver cells," Biomed. Microdevices, vol. 9, no. 5, pp. 637-645, Oct. 2007.

[44] Y. C. Fung, Biomechanics: Mechanical Properties of Living Tissues, 2nd ed. New York: Springer-Verlag, 1993.

[45] C. E. Lentner, Geigy Scientific Tables. Basle, Switzerland: Ciba-Geigy, 1981. 
[46] S. B. McKenzie, Clinical Laboratory Hematology. Upper Saddle River, NJ: Prentice-Hall, 2004.

[47] H. M. Shapiro, E. R. Schildkraut, R. Curbelo, C. W. Laird, R. B. Turner, and T. Hirschfeld, "Combined blood cell counting and classification with fluorochrome stains and flow instrumentation," J. Histochem. Cytochem., vol. 24, no. 1, pp. 396-411, Jan. 1976.

[48] S. Ben-Sasso, D. Patinkin, N. B. Grover, and F. Doljansk, "Electrical sizing of particles in suspensions. IV. Lymphocytes," J. Cell. Physiol., vol. 84, no. 2, pp. 205-214, Oct. 1974.

[49] M. A. Van Dilla, M. J. Fulwyler, and I. U. Boone, "Volume distribution and separation of normal human leucocytes," Proc. Soc. Exp. Biol. Med., vol. 125, no. 2, pp. 367-370, Jun. 1967.

[50] N. Darnton, O. Bakajin, R. Huang, B. North, J. O. Tegenfeldt, E. C. Cox, J. Sturm, and R. H. Austin, "Hydrodynamics in 2-1/2 dimensions: Making jets in a plane," J. Phys., Condens. Matter, vol. 13, no. 21, pp. 4891-4902, May 28, 2001.

[51] E. E. Michaelides, "Review-The transient equation of motion for particles, bubbles, and droplets," Trans. ASME, J. Fluids Eng., vol. 119, pp. 233-247, Jun. 1997.

[52] P. A. Aarts, S. A. van den Broek, G. W. Prins, G. D. Kuiken, J. J. Sixma, and R. M. Heethaar, "Blood platelets are concentrated near the wall and red blood cells, in the center in flowing blood," Arterioscler. Thromb. Vasc. Biol., vol. 8, no. 6, pp. 819-824, Nov./Dec. 1988.

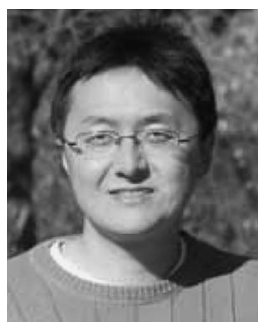

Siyang Zheng received the B.S. degree in biological sciences and biotechnologies from Tsinghua University, Beijing, China, in 1996, the M.S. degree in electrical engineering from Pennsylvania State University, University Park, in 2000, and the Ph.D. degree in electrical engineering from the California Institute of Technology (Caltech), Pasadena, in 2007 under the guidance of Dr. Yu-Chong Tai.

He was with Lucent Technologies, Holmdel, NJ, for one year before he joined the Micromachining Group, Caltech, as a graduate student in 2002. He is currently with Caltech as a Postdoctoral Scholar. His broad research experiences have covered lab-on-a-chip systems for cell separation and analysis, electrical impedance sensing, laser-induced fluorescence detection, MEMS sensors/actuators, microfabrication/nanofabrication, on-chip molecular selfassembly, and microfluidic integration technology. He has authored more than 20 published refereed journal/conference papers and has filed several industrial patents over the years.

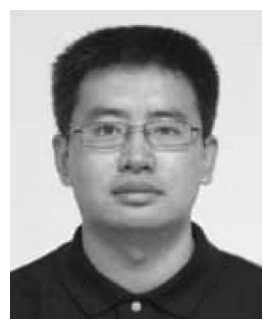

Jing-Quan Liu received the B.S., M.S., and Ph.D. degrees in mechanical engineering from the Jilin University of Technology (now Jilin University), Changchun, China, in 1993, 1996, and 2000, respectively.

He was a Postdoctoral Research Associate in electrical engineering with Shanghai Jiao Tong University, Shanghai, China, from 2000 to 2002. During 1998-1999, he was a Researcher with Yamagata University, Yamagata, Japan. From February 2006 to January 2007, he was a Visiting Scholar in the Department of Electrical Engineering, California Institute of Technology, Pasadena. In 2002, he joined Shanghai Jiao Tong University, where he is currently a member of the faculty of the Institute of Micro/Nano Science and Technology. His research interests include MEMS, biomedical microsystems, and microfabrication processing technology.

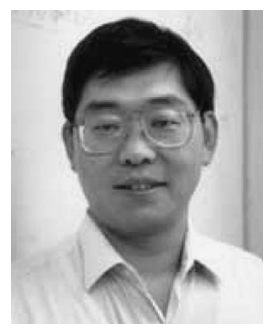

Yu-Chong Tai (M'97-SM'03-F'06) received the B.S. degree in electrical engineering from National Taiwan University, Taipei, Taiwan, R.O.C., in 1981, and the M.S. and Ph.D. degrees in electrical engineering from the University of California, Berkeley, in 1986 and 1989, respectively.

$\mathrm{He}$ is a member of the faculty with the Department of Electrical Engineering, California Institute of Technology (Caltech), Pasadena, where he built the Caltech Micromachining Laboratory and currently is a Professor of electrical engineering, mechanical engineering, and bioengineering. His current research interests include flexible microelectromechanical systems (MEMS), bioMEMS, MEMS for retinal implants, parylene-based integrated microfluidics, neuroprobes/neurochips, and high-performance liquid chromatography (HPLC)-based laboratory-on-a-chip systems.

Dr. Tai is the recipient of several awards, including the IBM Fellowship, the Best Thesis Award, the Presidential Young Investigator Award, and the David and Lucile Packard Fellowship. He was the Cochairman of the IEEE International MEMS 2002 Conference, Las Vegas, NV. 